\title{
Localization-delocalization transition in a presence of correlated disorder: The random dimer model
}

\author{
Tigran Sedrakyan* \\ ITP, University of Bern, Sidlerstrasse 5, CH-3012, Bern, \\ Switzerland and Yerevan Physics Institute, Br. Alikhanian str.2, Yerevan 36, Armenia
}

(Dated: October 31, 2018)

\begin{abstract}
The one dimensional dimer model is investigated and the localization length calculated exactly. The presence of delocalized states at $E_{c}=\epsilon_{a, b}$ of two possible values of the chemical potential in case of $\left|\epsilon_{a}-\epsilon_{b}\right| \leq 2$ is confirmed and the corresponding indices of the localization length were calculated. The singular integral equation connecting the density of states with the inverse of the localization length is solved and the analytic expression for the density of states compared with the numerical calculations.
\end{abstract}

After Anderson's works [1], 2] it became clear that all states of the systems in one or two dimensional spaces, which are putted into the fully disordered potential field (independent site-energy disordered Anderson model), are exponentially localized. In [3]- $[\underline{6}]$ it has been shown, that this claim is true for any strength of disorder and the localization is present in less than three dimensions even for an infinitesimal amount of full disorder. The idea arises that the type of disorder underlies of localizationdelocalization (insulator-metal) transition of one dimensional systems and in order to understand its nature we need to investigate the conditions, under which delocalized states can appear. That is why it is necessary to consider experimentally [25] and theoretically [7]- 24] one-dimensional systems with short(or long)-range correlated disorder, where the random variables are not fully independent, but are correlated at short (long) distances. The vanishing of the localization and the appearance of diffusion of electrons by correlations was further put forward for the explanation of high conductivity of polymers such as doped polyaniline, which can be approached by random-dimer model [8], 11]. The transport properties of random semiconductor superlattices also exhibit [23, 24, 25] delocalization in case of correlated disorder.

One of the simplest tight-binding and numerically best studied model with the nearest-neighbor correlated disorder is the random-dimer model [9]-[14], where one (or both) of the two possible values of site potentials $\epsilon_{a}$ and $\epsilon_{b}$ are random in pairs and appearing with probabilities $p$ and $1-p$. In these papers authors have analyzed the mentioned model and it was shown numerically that initially localized electron can become delocalized if $\left|\epsilon_{a}-\epsilon_{b}\right| \leq 2 t$ (here $t$ is a constant electron hopping coefficient). In the paper 15. the authors have studied the dimer model by numerical and semi-analytical methods. In case when both site potentials appear in pairs, they have calculated the critical energies coinciding with results of $[\underline{9}]$, and correlation length index $\nu=2$ (superdiffusion) when $\left|\epsilon_{a}-\epsilon_{b}\right|<2 t$, and equal to 1 for $\left|\epsilon_{a}-\epsilon_{b}\right|=2 t$. They present also some calculations of the density of states and the correlation length indices for different values $p$ for the probability. In the papers [16], [17] similar results were obtained by use of numerical methods. An interesting analytic approach was developed in [18].

Since in all papers above the calculations were done mainly numerically (though there are some analytical arguments supporting the presence of delocalized point), the question whether the correlation length is infinite at some point (critical point) or finite and equal to lattice size (extended state), remains open. In order to answer this questions it is reasonable to calculate the critical energy, the correlation length, the correlation length index and the density of states of paired-dimer model analytically and compare them with already obtained numerical results. Our aim in this article is to fill this gap and, by use of technique developed in 23] and 24], investigate the physical quantities mentioned above analytically.

In this article we will concentrate on one-dimensional tight-binding model of random binary alloy with on site potential field $\epsilon_{a}, \epsilon_{b}$, which are assigned randomly on the lattice sites with probabilities $p$ and $(1-p)$ correspondingly. As it was mentioned above, the diffusion of electrons may occur if we introduce a short range correlation in the distribution of the site potentials. Here we focus on a particular realization of the dimer model, when the site potentials appear always in pairs. The example of certain lattice segment is .... $\epsilon_{a} \epsilon_{a} \epsilon_{a} \epsilon_{a} \epsilon_{b} \epsilon_{b} \epsilon_{a} \epsilon_{a} \epsilon_{b} \epsilon_{b} \epsilon_{b} \epsilon_{b} \epsilon_{a} \epsilon_{a} \ldots$, and it is clear that we have correlation in the probability distribution of nearest-neighbor site potentials.

We calculate the dimensionless Landauer resistance(correspondingly Landauer exponent) analytically and compare it with numerical simulations of Lyapunov exponent. In articles [20] Anderson and coauthors have argued, that the Lyapunov exponent is simply the half of Landauer one. Later, Kappus and Wegner have shown in 21] (confirmed in 22]), that the band center can be anomalous. In Figure 1 we present our result (for $\epsilon_{a}-\epsilon_{b}=t$ case) for the Lyapunov and the half of Landauer exponents. One can see the coincidence in a wide central region (and in the whole region in general) demonstrating that the Lyapunov and Landauer exponents have a same critical behavior. Exact analytic calculations of the Landauer resistance and the correlation length shows the existence of two real critical points with critical ener- 
gies $E_{\text {crit }}^{(1)}=\epsilon_{a}$, and $E_{\text {crit }}^{(2)}=\epsilon_{b}$ if $\left|\epsilon_{a}-\epsilon_{b}\right| \leq 2 t$. All other states are localized. We confirm the results of [9] and [15] in the region $\left|\epsilon_{a}-\epsilon_{b}\right|<2 t$ where there is a super-diffusion with the correlation length index $\nu=2$. However the situation is different in case $\epsilon_{a}-\epsilon_{b}=2 t$. Our analytical calculations show, that the correlation length index $\nu=1$ when approaching to the critical points $\epsilon_{a}$ and $\epsilon_{b}$ from inside, while $\nu=1 / 2$, when approaching from outside of the segment $\left[\epsilon_{a}, \epsilon_{b}\right]$. It seems to us that this fact was not observed in the earlier works.

We have analyzed also the density of states. In article 28] D.Thouless has found a singular integral equation connecting the density of states with the Lyapunov exponent. By differentiating this equation(which is Carlemans equation) one can reduce it to Hilbert transform problem. In order to find a solution by expressing the density of states via the derivative of the Lyapunov exponent we have used the theory of singular integral equations presented in the book by Muskhelishvili [29]. In a class of functions which have a finite derivatives in all points the solution is defined by one arbitrary constant, which will be fixed by the condition that the integral of density of states is equal to one. We are presenting this solution with the use of half of Landauer exponent and compare it with the numerical calculation (which have been also presented earlier in [15]). Again, we found an excellent correspondence of the two results in the central critical region. The numerical data exhibits strong fluctuations at the edges of the energy region due to luck of enough large $N$ size of thermodynamic limit. But if one will allow the presence of points where the derivative of the density of states is infinite, then one can find other solutions. Unfortunately present numerical calculations are not allowing to answer to this question precisely, but it is clear, that by fixing the number and places of the singular points we can find a unique solution for the density of states.

The Schrödinger equation for the stationary eigenstates $\psi_{i}(E)$ of the eigenenergy $E$ is

$$
\left(E-\epsilon_{i}\right) \psi_{i}-\psi_{i+1}-\psi_{i-1}=0, \quad i=1,2, \ldots, N
$$

where $\epsilon_{n}$ is the chemical potential at the site $n$ (it can be regarded also as an external potential), $N$ is the number of atoms in the system and we have re-scaled the energies by the hopping parameter $t$. Let us define $\epsilon_{a}-\epsilon_{b}=m$.

The Schrödinger equation (11) can be written via the $2 \times 2$ Transfer matrix $T_{i}$ as follows

$$
\left(\begin{array}{l}
\psi_{i+1} \\
\psi_{i}
\end{array}\right)=\left(\begin{array}{cc}
E-\epsilon_{i} & -1 \\
1 & 0
\end{array}\right)\left(\begin{array}{l}
\psi_{i} \\
\psi_{i-1}
\end{array}\right) \equiv T_{i}\left(\begin{array}{l}
\psi_{i} \\
\psi_{i+1}
\end{array}\right) .
$$

One can easily find out following property $T_{i}^{-1}=\sigma_{2} T_{i}^{\dagger} \sigma_{2}$ ( $\sigma_{2}$ is Pauli matrix), which means that $T_{i}$ is an element of the group $S U(1,1)$. By iterating this equation we can relate $\left(\psi_{i+1}, \psi_{i}\right)$ and $\left(\psi_{0}, \psi_{1}\right)$ :

$$
\left(\begin{array}{l}
\psi_{i+1} \\
\psi_{i}
\end{array}\right)=\prod_{k=i}^{1} T_{k}\left(\begin{array}{l}
\psi_{1} \\
\psi_{0}
\end{array}\right) \equiv M_{i}\left(\begin{array}{l}
\psi_{1} \\
\psi_{0}
\end{array}\right)
$$

where the product $M_{N}=\prod_{i=N}^{1} T_{i}$ is the total Transfer matrix of the system of $N$ unit cells.

Oseledec's theorem [26] states that the eigenvalues of following matrices

$$
\Gamma_{N}=\left(M_{N} M_{N}^{\dagger}\right)^{1 / 2 N},
$$

have a limit $e^{\bar{\gamma}}$ with nonnegative $\bar{\gamma}$, which called Lyapunov exponent. Due to self-averaging property $\bar{\gamma}$ is equal to $<\log \Gamma>$. This quantity is very hard to calculate directly. Instead, the calculation of the matrix $G_{N}=M_{N} \otimes M_{N}^{\dagger}$ and its disorder average can be done exactly [23]. The dimensionless Landauer resistance $\rho=<G_{12,21}>$ is defined by 12,21 matrix element of $G$.

By use of the formula for decomposition of direct product of two spin- $1 / 2$ states into the direct sum of scalar and spin- 1 states, we can decompose the direct product of $T_{j}$ and $T_{j}^{-1}$ matrices into scalar and spin-1 parts as

$$
\left(T_{j}\right)_{\alpha^{\prime}}^{\alpha}\left(T_{j}^{-1}\right)_{\beta}^{\beta^{\prime}}=\frac{1}{2}(\delta)_{\beta}^{\alpha}(\delta)_{\alpha^{\prime}}^{\beta^{\prime}}+\frac{1}{2}\left(\sigma^{\mu}\right)_{\alpha^{\prime}}^{\beta^{\prime}} \Lambda_{j}^{\mu \nu}\left(\sigma^{\nu}\right)_{\beta}^{\alpha},
$$

where $\sigma^{\nu}, \quad \nu=1,2,3$ are Pauli matrices. In this expression the Kronecker $\delta$ 's define the scalar part, while

$$
\Lambda_{j}^{\mu \nu}=\frac{1}{2} \operatorname{Tr}\left(T_{j} \sigma^{\mu} T_{j}^{-1} \sigma^{\nu}\right)
$$

defines the spin-1 part. By multiplying the expression (5) from the left and right hand sides by $\sigma_{2}$ we will have

$$
\left(T_{j}\right)_{\alpha^{\prime}}^{\alpha}\left(T_{j}^{+}\right)_{\beta}^{\beta^{\prime}}=\frac{1}{2}\left(\sigma_{2}\right)_{\beta}^{\alpha}\left(\sigma_{2}\right)_{\alpha^{\prime}}^{\beta^{\prime}}+\frac{1}{2}\left(\sigma^{\mu} \sigma_{2}\right)_{\alpha^{\prime}}^{\beta^{\prime}} \Lambda_{j}^{\mu \nu}\left(\sigma_{2} \sigma^{\nu}\right)_{\beta}^{\alpha} .
$$

As it was shown in article 23], the similar expression is correct for the total Transfer matrix $M_{N}$, but instead of $\Lambda_{j}^{\mu \nu}$ we will have a product $\prod_{j=1}^{N} \Lambda_{j}$.

Now we should take into account the disorder and calculate the average of $\Gamma$ by random distribution of pairs of potentials $\left(\epsilon_{a}, \epsilon_{a}\right)$ and $\left(\epsilon_{b}, \epsilon_{b}\right)$.

$$
\langle G\rangle=\frac{1}{2} \sigma_{2} \otimes \sigma_{2}+\frac{1}{2}\left(\sigma^{\mu} \sigma_{2}\right) \otimes\left(\sigma_{2} \sigma^{\nu}\right)\left(\prod_{j=1}^{N / 2}\left\langle\Lambda_{j}^{2}\right\rangle\right)^{\mu \nu},
$$

where $3 \times 3$ matrix $\Lambda_{j}^{2}$ is defined as

$$
\left(\Lambda_{j}^{2}\right)^{\mu \nu}=\frac{1}{2} \operatorname{Tr}\left(T_{j}^{2} \sigma^{\mu}\left[T_{j}^{2}\right]^{-1} \sigma^{\nu}\right) .
$$

In the Dimer model under consideration we should average the square of $\Lambda_{i}$ as

$$
\Lambda=\left\langle\Lambda_{j}^{2}\right\rangle=p \Lambda_{j}^{2}\left(\epsilon_{a}\right)+(1-p) \Lambda_{j}^{2}\left(\epsilon_{b}\right),
$$


where the $\Lambda_{j}\left(\epsilon_{a}\right)$ and $\Lambda_{j}\left(\epsilon_{b}\right)$ are calculated for the site potentials $\epsilon_{a}$ and $\epsilon_{b}$ respectively.

Without loosing the generality one can choose $\epsilon_{a}=$ $-m / 2$ and $\epsilon_{b}=m / 2$. Then the resulting matrix $\Lambda$ will have the following elements:

$$
\begin{aligned}
\Lambda^{11} & =\left[2+\left((E-m / 2)^{2}-4\right)(E-m / 2)^{2}\right] \frac{1-p}{2} \\
& +\left[(E+m / 2)^{4}-4(E+m / 2)^{2}+2\right] \frac{p}{2}, \\
\Lambda^{12} & =\left((E-m / 2)^{2}-2\right)(E-m / 2)^{2} \frac{1-p}{2} \mathfrak{i} \\
& +(E+m / 2)^{2}\left((E+m / 2)^{2}-2\right) \frac{p}{2} \mathfrak{i}, \\
\Lambda^{13} & =\left((E-m / 2)^{2}-2\right)(E-m / 2)(p-1) \\
& -(E+m / 2)\left((E+m / 2)^{2}-2\right) p, \\
\Lambda^{21} & =\left((E-m / 2)^{2}-2\right)(E-m / 2)^{2} \frac{p-1}{2} \mathfrak{i} \\
& -(E+m / 2)^{2}\left((E+m / 2)^{2}-2\right) \frac{p}{2} \mathfrak{i}, \\
\Lambda^{22} & =\left((E-m / 2)^{4}+2\right) \frac{1-p}{2}+\left((E+m / 2)^{4}+2\right) \frac{p}{2}, \\
\Lambda^{23} & =(E-m / 2)^{3}(1-p) \mathfrak{i}+(E+m / 2)^{3} p \mathfrak{i}, \\
\Lambda^{31} & =\left((E-m / 2)^{2}-2\right)(E-m / 2)(1-p) \\
& +(E+m / 2)\left((E+m / 2)^{2}-2\right) p, \\
\Lambda^{32} & =(E-m / 2)^{3}(1-p) \mathfrak{i}+(E+m / 2)^{3} p \mathfrak{i}, \\
\Lambda^{33} & =1-2(E+m / 2)^{2}+4(E+m / 2) m(1-p) \\
& -2 m^{2}(1-p) .
\end{aligned}
$$

By the formula (3) $\psi_{N}=M_{12} \psi_{0}$ we have $\frac{\left|\psi_{N}^{2}\right|}{\left|\psi_{0}^{2}\right|}=M_{12} M_{21}^{+}$ and from

$$
\begin{aligned}
\left\langle M \otimes M^{+}\right\rangle_{12 ; 21} & =\frac{1}{2}\left[\left(\Lambda^{N / 2}\right)^{22}-\left(\Lambda^{N / 2}\right)^{11}-i\left(\Lambda^{N / 2}\right)^{12}\right. \\
& \left.+i\left(\Lambda^{N / 2}\right)^{21}\right] \simeq \lambda_{\max }^{N / 2}
\end{aligned}
$$

it is clear, that $\left\langle\frac{\left|\psi_{N}\right|^{2}}{\left|\psi_{0}\right|^{2}}\right\rangle \sim \lambda_{m a x}^{\frac{N}{2}}=e^{2 N \gamma}=e^{\frac{2 N}{\xi(E)}}$, where $\lambda_{\max }=e^{4 \gamma}=e^{\frac{4}{\xi(E)}}$ is the closest to unity eigenvalue of $\Lambda=\left\langle\Lambda_{j}^{2}\right\rangle$. Therefore the quantity $\rho=\left\langle M \otimes M^{+}\right\rangle_{12 ; 21}$, which is (see for example 28]) nothing but inverse module square of the Green function, defines the ratio of retarded over transmitted probabilities $\frac{|r|^{2}}{|t|^{2}}$ and can be regarded as the Landauer resistance. The closest to unity from above eigenvalue of $\left\langle\Lambda_{j}^{2}\right\rangle$ for an energy value $E$ defines the localization length $\xi=\frac{4}{\ln \lambda_{\max }}$ given in units of the length of the unit cell.

Strictly speaking the Lyapunov exponent $\bar{\gamma}$, defined by (44), differs from the Landauer $\gamma$. According to Anderson et al. 20] $\bar{\gamma}=\gamma / 2$. But, even when according to 21, 22] there is an anomaly, it is absolutely clear, that both exponents should define the same critical behavior(with the same critical index) at the same point $E_{c}$. Around that point they can differ only by the constant multiplicative factor, which is probably close to $1 / 2$. In Figure 1 . we

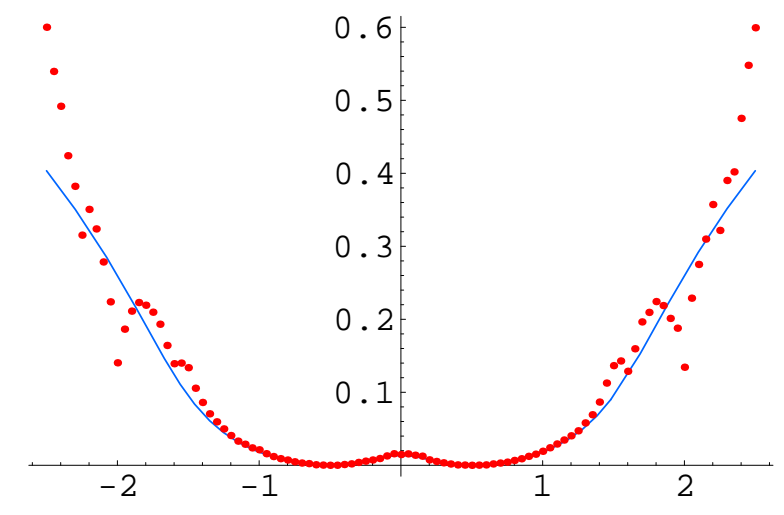

FIG. 1: $\mathrm{m}=1, \mathrm{p}=1 / 2$. Dots mark the Lyapunov exponent obtained by simulation of the chain of length 30000, while the curve is the half of Landauer exponent.

demonstrate the result of numerical simulations of Lyapunov exponent (dots), calculated iteratively in a standard way [27], and the half of Landauer exponent, which one can get from the formulas (11) and (12). We see perfect coincidence in the wide region around band center and in whole region in general.

The delocalized states are corresponding to the critical points $\xi\left(E_{c}\right)=\infty$ and can be found by the condition

$$
\begin{aligned}
& \operatorname{det}|i d-\Lambda|= \\
- & 2(E+m / 2)^{2}(E-m / 2)^{2} m^{2}(1-p) p=0
\end{aligned}
$$

and we see that $E_{c}= \pm m / 2$, which is in full accordance with the original numerical observations of the articles 7, 8, 10, 11].

One can find out the closest to unity eigenvalue $\lambda_{\max }$ by solving characteristic cubic equation for the matrix (11) and, therefore, calculate exactly the correlation length $\xi(E)=4 / \log [\lambda(E)]$.

For future considerations we will set $p=1 / 2$ for simplicity. Asymptotic-s of $\xi(E)$ around critical points $E_{c}= \pm m / 2$, which defines the correlation length indices $\nu=2$, are $\xi(E)=6 /(E+m / 2)^{2}$, (or $\left.6 /(E-m / 2)^{2}\right)$, for $m<2$ and

$$
\xi(E)= \begin{cases}\sqrt{2}(E+1)^{-1 / 2} & \text { for } E \rightarrow-1^{-} \\ 4(E+1)^{-1} & \text { for } E \rightarrow-1^{+} \\ 4(E-1)^{-1} & \text { for } E \rightarrow 1^{-} \\ \sqrt{2}(E-1)^{-1 / 2} & \text { for } E \rightarrow 1^{+}\end{cases}
$$

for $m=2$ respectively.

As we see, $\nu=2$ in the case $m<2$. For $m=2$ we see $\nu=1 / 2$, when we are approaching the critical points $E_{c}= \pm 1$ from the outside of the region $[-1,1]$, while $\nu=1$, when we are approaching them from the inside. One can check by direct calculations that these critical indices are independent from the choice of value of $p$.

Now let us analyze the density of states. According to article 28] the density of states $\rho(E)$ are connected with 
the Lyapunov exponent $\bar{\gamma}(E)$ as

$$
\bar{\gamma}(E)=f_{\mathcal{D}} \rho\left(E^{\prime}\right) \log \left[E^{\prime}-E\right] d E^{\prime},
$$

where $\mathcal{D}$ is the energy region of non-zero density of states and $f_{\mathcal{D}}$ should be understood as a Cauchy principal value. In our problem of dimers it is defined by the interval $-2-m / 2<E<2+m / 2$, because the kinetic energy on lattice can vary only in the interval $[-2,2]$.

Let us now differentiate the left and right hand sides of the equation (15) by $E$ and bring it to Hilbert's transform form

$$
\frac{d \bar{\gamma}(E)}{d E}=-f_{\mathcal{D}}\left(E^{\prime}\right) \frac{1}{E^{\prime}-E} d E^{\prime}
$$

The formula (14) shows that the cases $m=2$ and $m<2$ are essentially different. Therefore we will consider cases $m=1$ and $m=2$ separately.

In case of $m=1$, when we suppose that all derivatives of the $\rho(E)$ and $\gamma^{\prime}(E)$ functions are finite in the energy interval $[-2.5,2.5]$ beside the end points, according to [29] the general solution will be

$$
\begin{aligned}
\rho(E) & =\frac{1}{\pi \sqrt{2.5^{2}-E^{2}}} \\
& \cdot\left(\frac{1}{\pi} \int_{-2.5}^{2.5} \sqrt{2.5^{2}-\left(E^{\prime}\right)^{2}} \frac{d \bar{\gamma}\left(E^{\prime}\right) / d E^{\prime}}{E-E^{\prime}} d E^{\prime}+C\right) .
\end{aligned}
$$

It appears that $\int_{\mathcal{D}} \rho(E) d E=C$ and $C$ therefore should be set to be equal to one.

Since the Lyapunov exponent is hard to calculate analytically exactly lets us, following articles [20], put the half of Landauer exponent $\gamma(E) / 2=\frac{\ln \lambda_{\max }}{8}$ into the expression (17) and find the corresponding density of states. In the Fig.2 we present this solution for the case of $m=1$ together with the numerical calculation of the density of states, made for a chain of length $N=100$, averaged over random dimer $\epsilon$-s of the ensemble of 20000 samples. The numerical calculations were made by exact diagonalization of the Hamiltonian. As in the case of exponents (see Fig.1) we see very good correspondence of two curves in the energy region $E \in(-1.8,1.8)$, but numerical dates strongly fluctuate outside of that. This is the region, where the correlation length becomes of order of lattice size and where the Lyapunov exponent differs from the Landauer one. As it follows from the theory of singular integral equations [29] in case when $\rho(E)$ is infinite at some points one should divide whole energy band into segments with singular(and zero) points at the ends and write corresponding solution of the the Hilbert's problem
16. For simplicity we represent in Fig.2 the solution for case of absence singular points. But if one will be able to consider longer chain and observe in the numerical curve of $\rho(E)$ presence of singular points (one can see indication of that in Fig.2) then, following 29], it will be possible

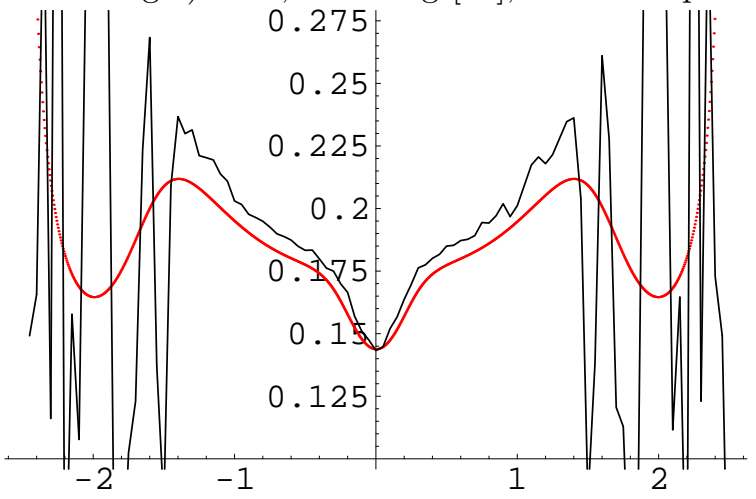

FIG. 2: The density of states versus energy for the case $\mathrm{m}=1$. Smooth curve represents the solution of the singular integral equation, while the fluctuating curve represents the numerical simulation for the ensemble of 20000 chain's of length $N=$ 100 .

to organize better fit of the numerical dates. It will not affect the good agreement of numerical simulations and analytic calculations on the basis of Landauer exponent in the most important central critical region.

The case of $m=2$ is more complicated. One can easily find out from expression (14), that the Landauer exponent $\gamma^{\prime}(E)$ has singular derivative at the points $E= \pm 1$ approaching to them from the outside. Therefore, first we should extract singular part from the $\gamma^{\prime}(E)$. Analyze shows, that the function $\chi(E)=\gamma^{\prime}(E)-\eta(E)$ with

$$
\eta(E)= \begin{cases}-\frac{2}{\sqrt{E^{2}-1}}, & \text { for } E \leq-1 \\ 0, & \text { for }-1 \leq E \leq 1 \\ \frac{2}{\sqrt{E^{2}-1}}, & \text { for } 1 \leq\end{cases}
$$

has no singular point. Therefore we can find the Hilbert transform for $\chi(E)$, add to it the Hilbert transform of $\eta(E)$ and obtain $\rho(E)$. Moreover, numerical calculations show also presence of some other singular points, in the region of energies $[-3,-1] \bigcup[1,3]$. Fig. 3 shows that the points $\pm 2, \pm 2.4$ looks singular, therefore, following the technique of solving singular integral equations presented in book 29] for the case of multiply connected region of integration, one can find the following expression for the density of states 


$$
\begin{aligned}
& \rho(E)=\frac{\sqrt{E^{2}-1.3^{2}} \sqrt{E^{2}-2.05^{2}} \sqrt{E^{2}-2.45^{2}}}{\pi \sqrt{E^{2}-3^{2}} \sqrt{E^{2}-2.4^{2}} \sqrt{E^{2}-2^{2}} \sqrt{E^{2}-1}} \\
& \cdot\left(\frac{1}{\pi} \int_{\mathcal{D}} \frac{\sqrt{\left(E^{\prime}\right)^{2}-3^{2}} \sqrt{\left(E^{\prime}\right)^{2}-2.4^{2}} \sqrt{\left(E^{\prime}\right)^{2}-2^{2}} \sqrt{\left(E^{\prime}\right)^{2}-1}}{\sqrt{\left(E^{\prime}\right)^{2}-1.3^{2}} \sqrt{\left(E^{\prime}\right)^{2}-2.05^{2}} \sqrt{\left(E^{\prime}\right)^{2}-2.45^{2}}} \frac{d \bar{\gamma}\left(E^{\prime}\right) / d E^{\prime}}{E-E^{\prime}} d E^{\prime}+\frac{i}{2}\right)+\frac{1}{2 \pi \sqrt{1-E^{2}}} \theta[E ;-1,1],
\end{aligned}
$$

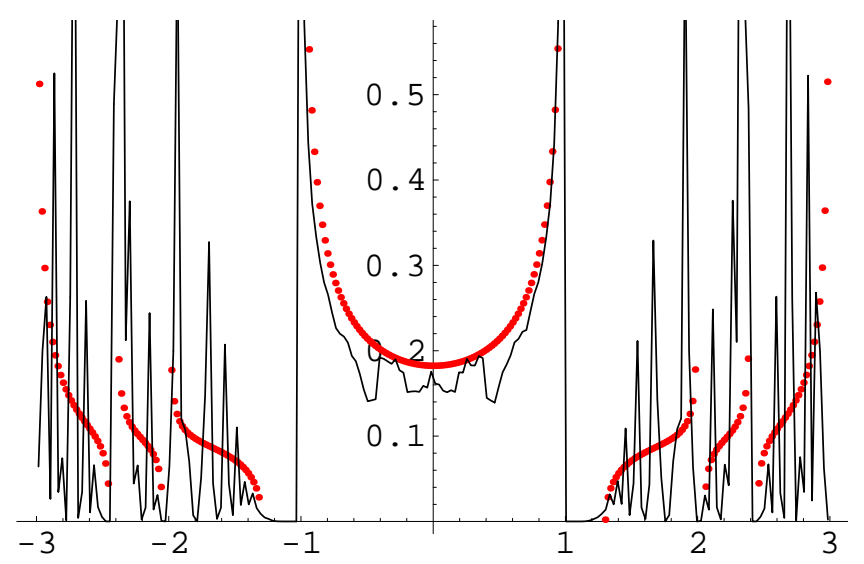

FIG. 3: The density of states versus energy for the case $m=2$. Dots represent the solution of the singular integral equation, while the fluctuating curve represents the numerical simulation for the ensemble of 20000 chain's of length $N=100$.

where $\mathcal{D}=[-3,-2.45] \bigcup[-2.4,-2.05] \bigcup[-2,-1.3][-1,1]$ $\bigcup[1.3,2] \bigcup[2.05,2.4] \bigcup[2.45,3]$ and the function $\theta[E ;-1,1]$ defined as follows

$$
\theta[E ;-1,1]=\left\{\begin{array}{lll}
1, & \text { if } & E \in[-1,1] \\
0, & \text { if } & E \notin[-1,1] .
\end{array}\right.
$$

This term appears in a result of Hilbert transform of $\eta(E)$.

In the Fig. 3 we present this solution defined by the half of Landauer exponent together with numerical results for $N=100$ and 20000 iterations. Since the half of Landauer exponent is in good agreement with the Lyapunov exponent in the center of energy band we expect good agreement between numerical and analytic results for the density of states there. Indeed, as in case of $m=1$ we see, that the analytic curve fits the numerical results in the region $E \in[-1,1]$ with $1 \%$ accuracy. We see also strong fluctuations of numerical density of states at the corners of the region $[-3,3]$. In case of better numerical results (made for much larger $\mathrm{Ns}$ ) one could define precisely whether there are more singularities of the derivative of $\rho(E)$ or not, choose an appropriate solution of the singular integral equation and organize better fit.

Conclusions: By use of technique developed in 23, 24. we have calculated analytically exactly the Landauer resistance and the corresponding exponent in the random dimer model. We have analytically observed presence of delocalization transition for $m \leq 2$ confirming results obtained earlier. Corresponding critical indices have been found. For $m<2$ we have obtained $\nu=2$. For limiting value $m=2$ we have obtained asymmetric behavior for the localization length when approaching to critical values $E_{c}= \pm 1$ from the left and right hand sides. It appeared, that $\nu=1 / 2$ when approaching to boundaries of the segment $[-1,1]$ from outside and $\nu=1$ - from inside. It looks that this fact was not observed earlier.

We compare the half of Landauer exponent with the numerical calculations of the Lyapunov exponent (Fig.1) and found good correspondence in the central region in accordance with [20].

By use of theory of singular integral equations 29] we found analytic solution of the Thouless equation (15) for the density of states. Instead of Lyapunov exponent we put the half of Landauer exponent into the expression of the density of states and compare it with numerical calculations made by the diagonalization of the Hamiltonian. As it was expected we got good correspondence (1\%) in the central region. The fit of numerical results at the boundaries can be improved by considering longer chain and choosing solution of singular integral equations for multiply connected regions.

Acknowledgment: I am grateful to A.Sedrakyan for the formulation of the problem and support, P. Hasenfratz for many valuable discussions and remarks and T.Hakobyan for the help in numerical analyzes. I also would like to acknowledge the ITP of Bern University for hospitality, where the major part of this work was done.

This work was supported in part by SNF SCOPE grant, Volkswagen foundation and the INTAS grant 00561.

* Electronic address: tigrans@moon.yerphi.am

[1] P.W. Anderson, Phys. Rev. 109,1492(1958).

[2] R.Abou-Chacra, P.W.Anderson, D.J.Thouless, J.Phys.C 6,1734(1973).

[3] N. F. Mott, D. Davis, Electronic Processes in NonCrystalline Materials (Clarendon, Oxford 1979).

[4] J. M. Ziman, Models of Disorder, Cambridge University Press, London 1979.

[5] I. M. Lifshitz, S. A. Gredeskul, L. A. Pasture, Introduction to the Theory of Disordered Systems, (Wiley, NY 
1988).

[6] K. Ishii, Suppl. Prog. Theor. Phys. 53,77(1973).

[7] J. C. Flores, J. Phys. Condens. Matter 1, 8471(1989).

[8] P. Phillips, H.-L. Wu, Science 252,1805(1991).

[9] D. H. Dunlap, H.-L.Wu, P.Phillips, Phys.Rev.Lett.65, 88 (1990).

[10] H.-L.Wu and P.Phillips, J.Chem.Phys.93,7369(1990).

[11] H.-L.Wu and P.Phillips, Phys.Rev.Lett. 66,1366(1991).

[12] A. Bovier, J. Phys. A 25, 1021 (1992).

[13] M. Hilke, J.C. Flores, Phys. Rev. B 55,10625 (1997). M. Hilke, J. of Phys. A: Math.Gen. 30,L367 (1997).

[14] H.-L. Wu, W. Goff, and P. Phillips, Phys. Rev. B 45, 1623 (1992).

[15] S. N. Evangelou, A.Z.Wang, Phys.Rev.B47,13126(1993).

[16] P.K.Datta,D.Giri,K.Kundu, Phys.Rev.B47,10727(1993).

[17] P.K.Datta,D.Giri,K.Kundu, Phys.Rev.B48,16347(1993).

[18] F.M.Izrailev,A.A.Krokhin, Phys.Rev.Lett.B82,4062(1999), L. Tessieri, F. M. Izrailev, Physica E9,405 (2001).

[19] I.Varga, J.Pipek, J.Phys.:Condens.Matter10,305(1998).
[20] P. Anderson, D. Thouless, E. Abrahams, D. Fisher, Phys.Rev.B 22,3519 (1980),

P. Anderson, Phys.Rev.B 22,4828 (1981).

[21] M. Kappus, F. Wegner, Z.Phys.B 45,15 (1981).

[22] B. Derrida, E. Gardner, J.Physique 45,1283 (1984).

[23] D.Sedrakyan, A.Sedrakyan, Phys.Rev.B60,10114 (1999).

[24] F. Dominguez-Adame, I. Gomez, A. Avakyan, D. Sedrakyan, A. Sedrakyan, Pys.Stat.Sol.(b) 221,633 (2000).

[25] V. Bellani, E. Diez, R. Hey, L. Toni, L. Tarricone, G. B. Parravincini, F. Dominguez-Adame, R. Gomes-Alcala, Phys. Rev. Lett. 82,2159 (1999).

[26] V. I. Oseledec, Trans. Moscow Math. Soc. 19,197 (1968).

[27] A. MacKinnon, J. Phys. C13, L1031 (1980), A. MacKinnon, B. Kramer, Z.Phys.B-Condenced Matter $\mathbf{5 3}, 1$ (1983).

[28] D.Thouless, J. Phys. C: Solid State Phys. 5,77 (1972).

[29] N. Muskhelishvili, Singular Integral Equations, Moscow 1946. 